\title{
FIXED SUBALGEBRA OF A FROBENIUS ALGEBRA
}

\author{
KAI WANG
}

\begin{abstract}
In this note, we study the fixed subalgebra of a Frobenius algebra under a finite group of automorphism. If the automorphisms preserve the nondegenerate bilinear form. then the fixed subalgebra is also Frobenius.
\end{abstract}

1. Introduction. Let $F$ be a fixed field of characteristic $p$ including the case $p=0$ and let $A$ be a finite-dimensional Frobenius algebra over $F$. Let $G$ be a finite group of algebraic automorphisms of $A$, i.e. automorphisms that fix the ground field. and let $A^{(i}$ be the fixed subalgebra under $G$. It has been shown [3] by J. L. Pascaud and J. $V$ alette that it does not necessarily follow that $A^{(j}$ is Frobenius. In a recent paper [1]. G. Azumaya has shown that if $B$ is a finite-dimensional commutative algebra generated by a single element and if $A=B \otimes B$, then the fixed subalgebra of $A$ under the involution $x \otimes y \rightarrow y \otimes x$ is Frobenius if and only if $p \neq 2$ or $B$ is separable. On the other hand, by definition [2] a finite-dimensional algebra $A$ with identity is Frobenius if and only if there is a nondegenerate associative bilinear form on $A$. The purpose of this note is to study the fixed subalgebra of an arbitrary Frobenius algebra under a finite group of algebraic automorphisms which preserves the bilinear form. Our main result is the following.

THEOREM 1.1. Let $A$ be a finite-dimensional Frobenius algebra with a nondegenerate associative bilinear form $\varphi$. Let $G$ be a finite group of algebraic automorphisms of $A$ such that $\varphi(g x, g v)=\varphi(x, y)$ for all $x, y \in A, g \in G$. Then the fixed subalgebra $A^{G}$ is Frobenius provided $p \nmid|G|$.

As an application, we will prove the following corollary which generalizes Azumaya's result.

COROLlaRY 1.2. Let $B$ be a finite-dimensional Frobenius algebra over $F$ and let $A=B \otimes B$. Let $\tau$ be the involution on $A$ which is defined by $\tau(x \otimes y)=y \otimes x$. Then the fixed subalgebra $A^{G}$ is also Frobenius provided $p \neq 2$.

2. Bilinear forms of a vector space. In this section, let $E$ be an $n$-dimensional vector space over $F$ and let $\left\{l_{1}, \ldots, l_{n}\right\}$ be a fixed basis for $E$. For a set of vectors $\left\{x_{1}, \ldots, x_{m}\right\}$, we use $\left\langle x_{1}, \ldots, x_{m}\right\rangle$ to denote the subspace generated by $\left\{x_{1} \ldots, x_{m}\right\}$. Since the proofs are easy, the following lemma and propositions are quoted without proofs.

Received by the editors March 3, 1982 and, in revised form. August 12, 1982. 1980 Mathematics Subject Classification. Primary 16A36, 16A74.

(c)1983 American Mathematical Society $0002-9939 / 82 / 0000-0825 / \$ 01.50$ 
LEMMA 2.1. Let $\varphi$ be a bilinear form on $E$. Then $\varphi$ is nondegenerate if and only if the $n \times n$ matrix $\left[\varphi\left(l_{i}, l_{j}\right)\right]$ is nonsingular.

Proposition 2.2. Let $\varphi$ be a bilinear form on $E$ and let $\left\{x_{1}, \ldots, x_{m}\right\}$ be a set of vectors. Then the restriction of $\varphi$ to $\left\langle x_{1}, \ldots, x_{m}\right\rangle$ is nondegenerate if and only if $\operatorname{rank}\left[\varphi\left(x_{i}, x_{j}\right)\right]=\operatorname{dim}\left\langle x_{1}, \ldots, x_{m}\right\rangle$.

Proposition 2.3. For $i=1,2$, let $\varphi_{i}$ be a nondegenerate bilinear form on vector spaces $E_{1}$. Then the tensor product $\varphi=\varphi_{1} \otimes \varphi_{2}$ is also nondegenerate.

3. A key proposition. As in $\S 2$, let $E$ be an $n$-dimensional vector space over $F$. Let $G$ be a finite group of automorphisms of $E$ and let $E^{G}=\{x \in E \mid g x=x$ for all $g \in G\}$. We will always assume that $p \nmid|G|$. Again, we leave the proof of the following lemma to the readers.

LEMMA 3.1. Let $\tau$ be an endomorphism of $E$ which is defined by $\tau x=(1 /|G|) \sum_{g \in G} g x$. Then $E^{G}=\operatorname{Im} \tau$.

Let $\varphi$ be a bilinear form on $E$. Recall that an automorphism $g$ of $E$ is said to be an automorphism of $\varphi$ if $\varphi(g x, g y)=\varphi(x, y)$ for all $x, y \in E$. The following proposition is the key in our study.

THEOREM 3.2. Let $\varphi$ be a nondegenerate bilinear form on $E$ and let $G$ be a finite group of automorphisms of $\varphi$. If $p \nmid|G|$ then the restriction of $\varphi$ to $E^{(i)}$ is also nondegenerate.

Proof. Let $\left\{l_{1}, \ldots, l_{n}\right\}$ be a basis for $E$ and let $\tau l_{l}=\sum_{k=1}^{n} a_{1 k} l_{k}$. By Lemma 3.1. $\left\langle\tau l_{1}, \ldots, \tau l_{n}\right\rangle=E^{(i}$.

$$
\begin{aligned}
& {[\varphi(\tau l, \tau l,)]=\left[\varphi\left(\frac{1}{|G|} \sum_{g \in G ;} g l_{i} \cdot \frac{1}{|G|} \sum_{h \in(;} h l\right)\right]} \\
& =\left[\frac{1}{|G|^{2}} \sum_{g \in G} \sum_{h \in G} \varphi\left(g l_{i}, h l_{j}\right)\right]=\left[\frac{1}{|G|^{2}} \sum_{h \in G} \sum_{g \in G ;} \varphi\left(h^{-1} g l_{l}, l_{l}\right)\right] \\
& =\left[\frac{1}{|G|} \sum_{g \in C} \varphi\left(g l_{1}, l_{,}\right)\right]=\left[\varphi\left(\tau l_{1}, l_{,}\right)\right]=\left[\varphi\left(\sum_{k=1}^{n} a_{i k} l_{k}, l\right)\right] \\
& =\left[\sum_{k=1}^{n} a_{1 k} \varphi\left(l_{k}, l_{1}\right)\right]=\left[a_{1},\right]\left[\varphi\left(l_{1}, l_{,}\right)\right] \text {. }
\end{aligned}
$$

Note that $[\varphi(l, l)$,$] is nonsingular since \varphi$ is nondegenerate. This implies that

$$
\operatorname{rank}\left[\varphi\left(\tau l_{1}, \tau l,\right)\right]=\operatorname{rank}\left[a_{1,}\right]=\operatorname{dim} \operatorname{Im} \tau=\operatorname{dim} E^{(i} .
$$

It follows from Proposition 2.2 that $\left.\varphi\right|_{E^{\prime}}$, is nondegenerate. 
4. Proofs of Theorem 1.1 and Corollary 1.2. It is clear that Theorem 1.1 follows immediately from Theorem 3.2 and the definition of Frobenius algebra.

For Corollary 1.2 note that if $\psi$ is a nondegenerate associative bilinear form on $B$, then $\varphi=\psi \otimes \psi$ is a nondegenerate associative bilinear form on $A=B \otimes B$. Then

$$
\begin{aligned}
\varphi\left(\tau(x \otimes y), \tau\left(x^{\prime} \otimes y^{\prime}\right)\right) & =\varphi\left(y \otimes x, y^{\prime} \otimes x^{\prime}\right) \\
& =\psi\left(y, y^{\prime}\right) \psi\left(x, x^{\prime}\right)=\varphi\left(x \otimes y, x^{\prime} \otimes y^{\prime}\right) .
\end{aligned}
$$

It follows that $\tau$ is also an automorphism of $\varphi$. It follows that the fixed algebra $A^{\tau}$ is Frobenius.

\section{REFERENCES}

1. G. Azumava. Fived subalgehra of a commutatice Frobenius algehra. Proc. Amér. Math. Soc. 81 (1981). 213-216.

2. E. A. Behrens, Ring theory. Academic Press. New York. 1972.

3. J. L. Pascaud and J. Valette. Group acrions on Q-F rings. Proc. Amer. Math. Soc. 76 (1979). $43-44$

Department of Mathematics. Wayne State University. Detroit. Michigan 48202 